\title{
Shelterin protein keeps telomeres on edge
}

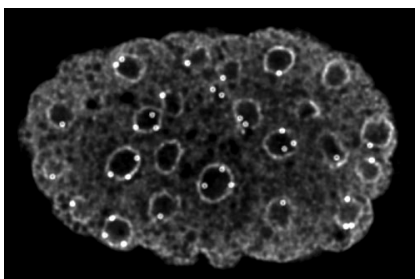

Telomeres (bright spots) are concentrated at the edges of nuclei in a nematode embryo.

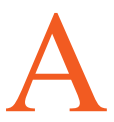

member of the shelterin complex helps moor telomeres at the nuclear periphery in worms, Ferreira et al. reveal.

Telomeres protect the ends of chromosomes, and the shelterin complex protects telomeres, preventing them from triggering the DNA damage response. Yeast cells shove

their telomeres to the fringe of the nucleus, and the marginalized structures gather into bunches. Plant cells also place their telomeres at the edge of the nucleus. Whether animal cells follow suit is unclear, because researchers have noted that telomeres only briefly remain at the nuclear periphery in cultured mammalian cells. Ferreira et al. zoomed in on nematode telomeres to address what happens during animal development.
The researchers discovered that worm telomeres also accumulate at the boundary of the nucleus, but, unlike in yeast cells, they don't cluster unless the worm lacks telomerase activity.

Embryos and larval worms rely on different mechanisms to anchor the telomeres at the rim of the nucleus, the team found. In embryonic worms, the shelterin subunit POT-1 helps position the telomeres. Two other proteins, the nuclear envelope protein SUN-1 and the SUMO ligase GEI-17, are also necessary. In larval worms, by contrast, telomeres remain in position even if one of these three proteins is missing. That discrepancy suggests that a different mechanism anchors telomeres in later developmental stages.

Why cells go to the trouble of arranging their telomeres at the nuclear rim isn't clear, but the positioning could indicate that genes near the ends of chromosomes have been silenced. Alternatively, the nuclear envelope may help shelterin protect chromosome ends from recombination.

Ferreira, H.C., et al. 2013. J. Cell Biol. http://dx.doi.org/10.1083/ jcb.201307181.

\section{Actin-capping protein lets epithelial cells take the pressure}

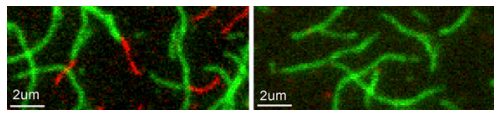

When CD2AP is absent (left), actin monomers (red) adhere to existing filaments (green), but addition of the protein (right) prevents this interaction.

y capping the tips of actin filaments, CD2AP reinforces the junctions between epithelial cells, Tang and Brieher show.

The actin cytoskeleton is a key structural element for intercellular junctions and dictates how tightly cells stick together. $\alpha$-Actinin- 4 is necessary for actin assembly at adherens junctions. However, the protein can't do the job alone.

Tang and Brieher identified a protein complex at adherens junctions that contains 10 to 15 members and then tracked down proteins that reside near $\alpha$-actinin- 4 in the complex. One of those neighbors was CD2AP. The researchers found that CD2AP caps Sip 1 liberates neural crest cells

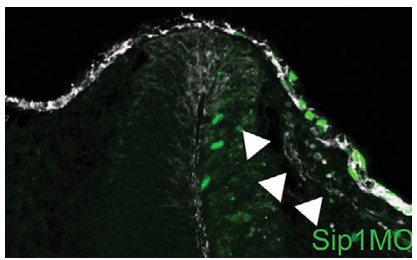

Neural crest cells sport more E-cadherin (white) on the right side of this embryo's neural tube, where researchers turned down Sipl production.

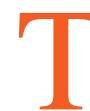
he protein Sip1 enables restless neural crest cells to leave home, Rogers et al. report.

From their birthplace in the embryonic neural tube, neural crest cells scatter throughout the body, giving rise to such diverse structures as the core of the adrenal gland and pigment cells in the skin. Neural crest cells share some similarities with metastatic cancer cells. Before they make a move, both types of cells undergo an epithelial-to-mesenchymal transition (EMT) in which they lose polarity, dramatically change morphology, and detach from their neighbors. The transcription factor Sip1 might help promote EMT in tumor cells, but its role in neural crest cells is uncertain. the barbed ends of actin filaments, preventing the attachment of actin monomers. The protein also prevented actin disassembly, Tang and Brieher showed.

To determine CD2AP's effects on adhesion, the researchers devised a technique for measuring the strength of cell layers. They put single layers of epithelial cells above a chamber containing fluid. Pumping more fluid into the chamber put more pressure on the cells. Low pressures had no effect on the epithelial layer, even if CD2AP was scarce. But at high pressures layers lacking CD2AP ripped, indicating that the adherens junctions between cells had failed. The researchers conclude that CD2AP stabilizes actin filaments and helps the cells cling to each other.

Tang, V.W., and W.M. Brieher. 2013. J. Cell Biol. http://dx.doi.org/10.1083/ jcb.201304143.

Rogers et al. found that neural crest cells in chick embryos also manufacture Sip1. After reducing Sip1 production in neural crest precursors, the researchers discovered that the cells left the neural tube but didn't travel far from it. They often remained stuck to other cells and showed reduced levels of the transcription factor SOX10, which marks migrating neural crest cells.

As cancer cells go through EMT, they replace E-cadherin molecules on their surface with $\mathrm{N}$-cadherin molecules. Rogers et al. found that Sip1 regulates the expression and localization of both adhesion molecules. Reducing Sip1 levels, the researchers determined, hiked the amount of E-cadherin in neural crest cells and reduced the amount of N-cadherin in the neural tube. The study shows that the migration of neural crest cells entails two separate steps - exit from the neural tube and dispersal. Sip1 is only necessary for the second step, which the protein might encourage by spurring the cells to decrease E-cadherin levels. Rogers, C.D., et al. 2013. J. Cell Biol. http://dx.doi.org/10.1083/jcb.201305050. 